Received: 20 July 2017

Accepted: 9 October 2017

Published online: 08 November 2017

\section{Global mapping of stratigraphy of an old-master painting using sparsity-based terahertz reflectometry}

\author{
Junliang Dong ${ }^{1,2}$, Alexandre Locquet ${ }^{2,1}$, Marcello Melis $\mathbb{1}^{3}$ \& D. S. Citrin ${ }^{1,2}$
}

The process by which art paintings are produced typically involves the successive applications of preparatory and paint layers to a canvas or other support; however, there is an absence of nondestructive modalities to provide a global mapping of the stratigraphy, information that is crucial for evaluation of its authenticity and attribution, for insights into historical or artist-specific techniques, as well as for conservation. We demonstrate sparsity-based terahertz reflectometry can be applied to extract a detailed 3D mapping of the layer structure of the 17th century easel painting Madonna in Preghiera by the workshop of Giovanni Battista Salvi da Sassoferrato, in which the structure of the canvas support, the ground, imprimatura, underpainting, pictorial, and varnish layers are identified quantitatively. In addition, a hitherto unidentified restoration of the varnish has been found. Our approach unlocks the full promise of terahertz reflectometry to provide a global and detailed account of an easel painting's stratigraphy by exploiting the sparse deconvolution, without which terahertz reflectometry in the past has only provided a meager tool for the characterization of paintings with paint-layer thicknesses smaller than $50 \mu \mathrm{m}$. The proposed modality can also be employed across a broad range of applications in nondestructive testing and biomedical imaging.

Studies at the intersection between the quantitative sciences and the humanities have been especially fruitful in the last few decades with the availability of new analytic tools that permit noninvasive studies of art. In particular, physical characterization of art using emerging tools may provide information that conclusively confirms or refutes judgments based on connoisseurship. Determination of the physical characteristics, not only across a painting but also in depth, is one of the most important procedures to gain insight into its structure. The layer-by-layer structure, or stratigraphy, reveals the sequential application of the preparatory and ground layers on the support (e.g., canvas or wood panel), pictorial layers, and varnish, as well as of possible subsequent revisions or restorations. A detailed knowledge of the stratigraphy provides a basis for evaluation of its authenticity and attribution, insight into historical or artist-specific techniques for art-historical studies, as well as the recognition of any decay and of consequent conservation and/or restoration requirements. For paintings, stratigraphic analysis can reveal the process in which the paint layers are applied, and consequently, to unveil how the artist worked. The conventional approach to obtain information on the stratigraphy is based on the characterization of the cross-sections of micro-samples taken from the objects with standard micro-analytical tools, such as visible-light and electron microscopy, energy-dispersive X-ray spectroscopy, Raman and infrared spectroscopy ${ }^{1}$. This approach is invasive, resulting in the destruction of the integrity of the painting.

Various noninvasive and noncontact modalities which can provide in-situ quantitative information in depth, such as confocal $\mathrm{x}$-ray fluorescence (with elemental distribution contrast) ${ }^{2}$, femtosecond pump-probe microscopy (with molecular and structural constrast) ${ }^{3}$, nuclear magnetic resonance (NMR) (with ${ }^{1} \mathrm{H}$ abundance constrast) ${ }^{4}$, and optical coherence tomography (with structural constrast) ${ }^{5}$, are under active research. Nonetheless, to date, the aforementioned optical techniques, as well as $\mathrm{x}$-ray fluorescence (the emitted photon is optical) have been limited to demonstrations permitting measurements to depths up to only tens or $\sim 200 \mu \mathrm{m}$ due to strong optical

${ }^{1}$ School of Electrical and Computer Engineering, Georgia Institute of Technology, Atlanta, GA, 30332-0250, USA. 2UMI 2958 Georgia Tech-CNRS, Georgia Tech Lorraine, 2 Rue Marconi, Metz, 57070, France. ${ }^{3}$ Profilocolore srl, Via Spluga, Roma, 2200 141, Italy. Correspondence and requests for materials should be addressed to J.D. (email: junliang.dong@gatech.edu) or D.S.C. (email: david.citrin@ece.gatech.edu) 
attenuation, while NMR has only been used to demonstrate several Z-scans across a painting ${ }^{6}$. These methods suffer from a set of overlapping difficulties for the task at hand, namely the global mapping of the stratigraphy of art paintings, including limited penetration as well as the unsuitability of sub-micrometric scale probes to provide the desired information on the 10- $\mu m$-to- $m m$ scales in depth and the 100- $\mu m$-to- $m$ scales in the transverse direction. In summary, while these techniques in some instances provide high-resolution depth information about paintings, they have not been demonstrated to enable access to produce a global 3D mapping of the stratigraphy of a painting.

Due to the penetrative capability of terahertz $(\mathrm{THz})$ electromagnetic radiation in broad classes of nonmetallic material ${ }^{7}$, THz reflectometry based on THz time-domain spectroscopy (THz-TDS) systems has attracted considerable interest for revealing the stratigraphy in situ, as well as hidden features of art paintings ${ }^{8}$, such as the artist's signature ${ }^{9}$, under-drawings ${ }^{10}$, and modifications due to the reuse of an earlier painting or canvas ${ }^{11}$. THz reflectometry may provide information in depth by analyzing the reflected $\mathrm{THz}$ signal with an incident approximately single-cycle $\mathrm{THz}$ pulse (which means that the spectrum of the $\mathrm{THz}$ radiation is broadband-from $\sim 100 \mathrm{GHz}$ up to $\sim 3 \mathrm{THz}$ for a typical THz-TDS system $)^{12}$. Due to dielectric discontinuities with depth associated with the various paint and other layers in the painting, reflected temporal $\mathrm{THz}$ echoes associated with the Fresnel coefficients at various interfaces are recorded as a function of transverse position in amplitude and time delay ${ }^{13}$. The stratigraphy of paintings can be reconstructed by precise extraction of $\mathrm{THz}$ echo parameters from the reflected $\mathrm{THz}$ signal. So far, some panel paintings and wall paintings have been studied by $\mathrm{THz}$ reflectometry, in which certain details of the stratigraphy have been revealed ${ }^{14-17}$. One lacuna in the past success of THz stratigraphic characterization of paintings, however, is the field of pre-19th century easel paintings ${ }^{18}$, where the paint layer thicknesses are usually smaller than $50 \mu \mathrm{m}^{19}$ (though paint-layer thickness varies by artist and style even throughout this period). This characteristic paint-layer thickness is optically thin in the THz regime, since it is much less than the time over which the $\mathrm{THz}$ pulse propagates within its duration, corresponding to the depth resolution of a typical $\mathrm{THz}_{\text {-TDS system }}{ }^{20}$. Nonetheless, there may be spectral information present at the relevant short wavelengths that is obscured in the raw signal. In the context of $\mathrm{THz}$ reflectometry, when dealing with optically thin paint layers, the $\mathrm{THz}$ echoes resulting from the various interfaces between layers will partially or even totally overlap in time and thus these echoes will merge rather than be distinct. Consequently, to our knowledge, the detailed stratigraphy of pre-19th century easel paintings has not been clearly revealed by $\mathrm{THz}$ reflectometry, as the paint layers in easel paintings are frequently very thin in the THz regime, especially for the 16th and 17th century easel paintings.

Deconvolution, which can yield sub-wavelength and sub-pulse-width depth resolution ${ }^{21}$, has shown great potential in resolving the overlapping echoes and characterizing the stratigraphy. The resulting impulse-response function provides a contrat mechanism for producing images that ideally depends on the sample structure rather than is dominated by characteristics of the probe itself. THz frequency-wavelet domain deconvolution (FWDD) has been specifically designed and has been applied to enhance the depth resolution and characterize multilayered structures with optically thin layers in the THz regime ${ }^{22,23}$. However, the depth resolution achieved by FWDD appears not to be sufficiently high to characterize the stratigraphy of typical easel paintings before the 19th century. In this study, in order to tease out the detailed stratigraphic information that is actually contained in the reflected $\mathrm{THz}$ signal, the sparse representation is introduced in the deconvolution process. Generally, the reflected $\mathrm{THz}$ signals from multilayered structures are a class of very special signals comprised of a limited number of echoes; therefore, the corresponding impulse-response functions have a sparse representation, which means that only a limited number of data points have non-zero values. This feature enables us to exploit the sparse constraint and retrieve the impulse-response function by sparsity-based time-domain deconvolution. Here we present $\mathrm{THz}$ reflectometry with subwavelength depth resolution for stratigraphic characterization based on a sparsity-based time-domain deconvolution algorithm as developed by our group ${ }^{24}$, which enables us to show the detailed stratigraphy of a 17th century easel painting based on $\mathrm{THz}$ reflectometry for the first time to our knowledge. The paint layers on the canvas support of this painting are significantly thinner than the dominant wavelength in the available $\mathrm{THz}$ spectrum; thus, the $\mathrm{THz}$ echoes reflected from the paint layers on the supporting canvas of this painting are strongly overlapped in the first cycle of the reflected $\mathrm{THz}$ signal. With the sparsity-based impulse-response function achieved by deconvolution, we gain 3D quantitative insight into the detailed stratigraphy above the canvas throughout the painting, including the varnish, pictorial layer, underpainting, imprimatura, and the ground layer. In addition, we identify delaminations in the pictorial layer associated with age-induced craquelure, and locate a hitherto unidentified extensive restoration of the painting. We emphasize, this is the first time, to our knowledge $\mathrm{THz}$ reflectometry has resolved multiple layers in an easel painting with such thin layers. Although we focus on easel paintings, the proposed modality can be applied to a wide range of culture heritage objects and provides invaluable information for art-historical studies, as well as potentially for conservation, restoration, and authentication.

\section{Results}

Sample and Experiment. The painting in this study was chosen as a typical painting for the period that is specifically of the type that has resisted considerable attempts to characterize its stratigraphy, i.e., the relevant layer thicknesses are on the order of tens of microns. It is a 17th century oil painting on canvas, entitled the Madonna in Preghiera attributed to the workshop of Giovanni Battista Salvi da Sassoferrato, in the collection of the Musée de la Cour d'Or, Metz--Metz Métropole, France, inventory number 11621, shown in Fig. 1(a). The dimensions of this painting are $24 \mathrm{~cm}$ by $32 \mathrm{~cm}$. The canvas is mounted on a wood stretcher. Visually, the paint application itself is smooth and uniform, though subsequently formed craquelure or other inclusions in the canvas give rise to nonuniform features in the surface texture. Multispectral imaging was performed with the Profilocolore HMI (Hypercolorimetric Multispectral Imaging) system, based on a modified Nikon D800FR camera to obtain the spectral reflectance of the surface in the range of $300-1000 \mathrm{~nm}$, with a spatial resolution of 


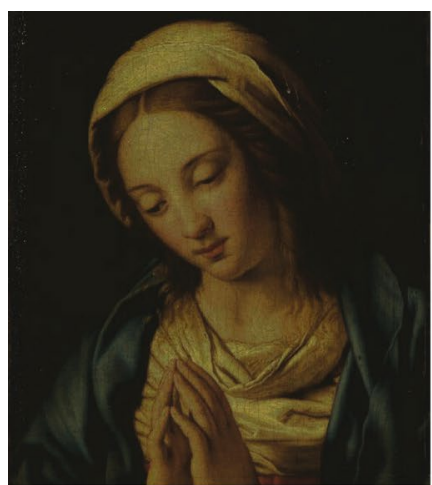

(a)

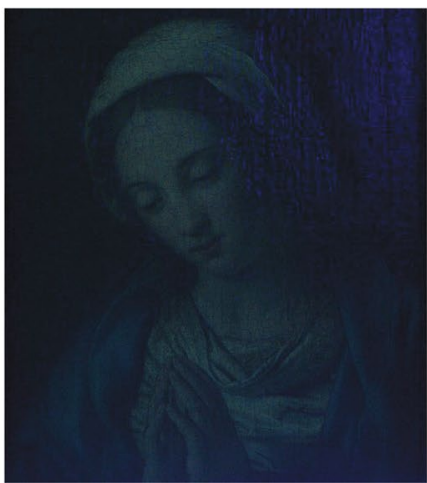

(b)

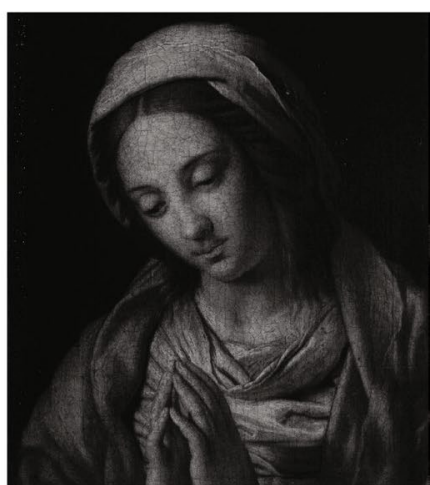

(c)

Figure 1. Images of Madonna in Preghiera. (a) Visible photograph of the Madonna in Preghiera; (b) Image of Madonna in Preghiera obtained by UV Fluorescence; (c) Image of Madonna in Preghiera obtained by IR Reflectography.

36 Mpixel. The intention is that different types of physical structures and pigments may be revealed by various wavelengths. Images obtained by ultraviolet (UV) fluorescence and infrared (IR) reflectography are shown in Fig. 1(b,c), respectively. UV fluorescence can reveal the presence of natural resin varnishes, which often fluoresce under UV light. It is also able to identify any retouchings on top of an aged varnish, since oil paint and newer varnish do not fluoresce under UV ${ }^{25}$. Retouchings therefore appear as dark patches on the varnish surface. In Fig. 1(b), a piece of dark patch near the Madonna's head can be clearly identified, which may corresponding to a retouching area. IR light can pass through the varnish and reveal the features of the surface and subsurface of the pictorial layers. In Fig. 1(c), age-induced craquelure in the pictorial layer can be observed.

$\mathrm{THz}$ reflective imaging based on a typical THz-TDS system (Teraview TPS Spectra 3000) was performed at almost normal incidence from the top (i.e., painted) surface of the painting. Before scanning the painting, a reflected $\mathrm{THz}$ reference signal was recorded by setting a metal plate at the sample position. The THz emitter and detector were raster-scanned by a set of motorized stages moving in the $X$ and $Y$ directions with a 1-mm step size over a $22 \mathrm{~cm}$ by $25 \mathrm{~cm}$ region of the painting. The painting was mounted paint facing downward supported by the edges of the stretcher in a horizontal configuration. After performing the scan, the positions of the last echo in the reflected signal at each point imaged, corresponding to the bottom surface (interface between the canvas and the air) of the painting, were aligned to the same position temporarily to correct for gravity-induced sag and convenience for further signal processing. After this alignment, a $3 \mathrm{D}$ volume raw data set was acquired.

THz Raw Images. First, we present the THz images based on the raw $\mathrm{THz}$ signals directly obtained from the scan. THz C-scans (two-dimensional presentation of data displayed as a top planar view of the painting) are shown in Fig. 2. For the THz C-scan in the time domain shown in Fig. 2(a), the imaging contrast mechanism chosen is the peak-to-valley amplitude of the reflected THz signal (mainly the peak-to-valley amplitude of the first cycle). The THz C-scan in the frequency domain can also be obtained by taking the Fourier transform of the raw waveform at each pixel, and integrating the magnitude of the frequency components in a given frequency window--between 0.5 and $1.0 \mathrm{THz}$, in the case of Fig. 2(b). THz C-scans based on these two contrast mechanisms mainly present the $\mathrm{THz}$ response of the paint pigments (as we clearly see that the dominant features are those of the visual aspects of the painting), and also reveal the surface roughness, as well as evidence of subsurface features. In Fig. 2(a), the Fresnel coefficient between the top-most paint layer and air depends on the refractive indices of the pigment, and so the image of the Madonna is clear. Many of the more irregular features, which will be commented on in depth below, are associated with the surface morphology of the painting. In the presence of a surface irregularity, enhanced scattering of $\mathrm{THz}$ radiation ensues, and consequently there is a weaker specular signal. In a similar fashion, Fig. 2(b) reveals similar (but not identical) features. In fact, the THz C-scan in the frequency domain is somewhat more effective in revealing surface features, as higher frequency components within the integrated spectral bandwidth, correspond to shorter wavelengths, bringing out small and subtle features.

While a THz C-scan is an $X, Y$ image of the painting, a THz B-scan (two-dimensional presentation displayed as a cross-sectional view of the painting) provides depth information along a line in the $X, Y$ plane. Note that optical delay in the received reflected signal serves as a surrogate for depth. Translating from optical delay to depth also requires a knowledge of the refractive indices of the various layers, which are assumed not to vary significantly within the $\mathrm{THz}$ spectral bandwidth. The $\mathrm{THz}$ B-scan based on the raw data with the cross-section $Y=150$ is plotted in Fig. 3 to show the structure of the painting. Note that in the present case, the contrast mechanism is simply the magnitude of the reflected signal at a given optical delay. The raw $\mathrm{THz}$ reflected signals at pixels (89, $150)$ and $(124,150)$ are also plotted for reference. These two signals show similar features associated with reflections off corresponding features, although there are slight differences due to local variations across the painting. The roughly horizontal features in the B-scan indicate reflections off interfaces between layers in the painting. The first large feature in optical delay (between 8 and 10 ps optical delay) is due to reflection off the painting surface, below which we see a clear feature ( $\sim 13 \mathrm{ps})$ due to the reflection off the ground-layer/canvas interface. The canvas layer can be clearly identified as corresponding to time delays from 13 to $20 \mathrm{ps}$. The pronounced horizontal 


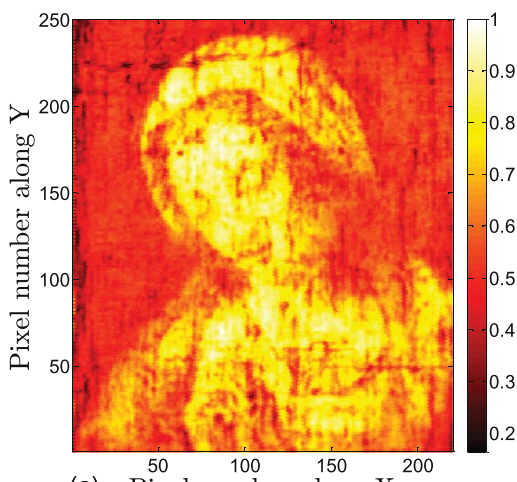

(a) Pixel number along $\mathrm{X}$

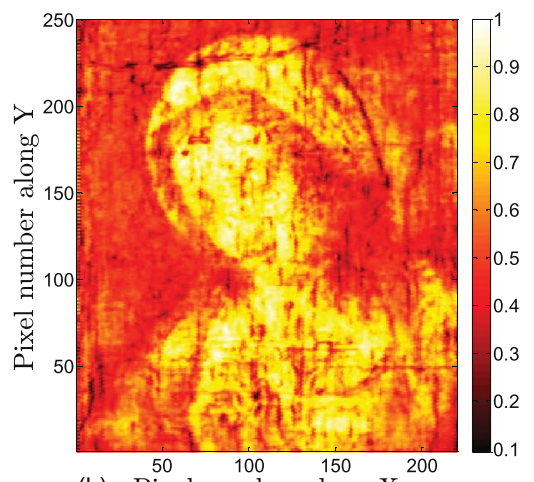

(b) Pixel number along $\mathrm{X}$

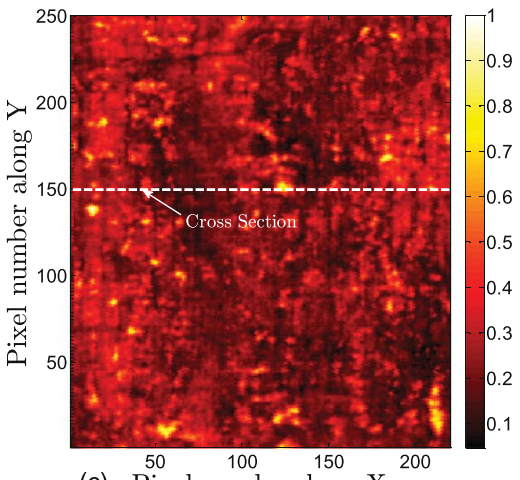

(c) Pixel numbe along $\mathrm{X}$

Figure 2. THz C-scans using on the raw $\mathrm{THz}$ data based on the following contrast mechanisms in the reflected signal: (a) peak-to-valley amplitude; (b) integrated spectrum between 0.5 and $1.0 \mathrm{THz}$; (c) peak-to-valley amplitude between 13 to 18 ps. The horizontal line at $Y=150$, marked 'cross section', is in reference to the B-scan in Fig. 3. Contrast is normalized to one.

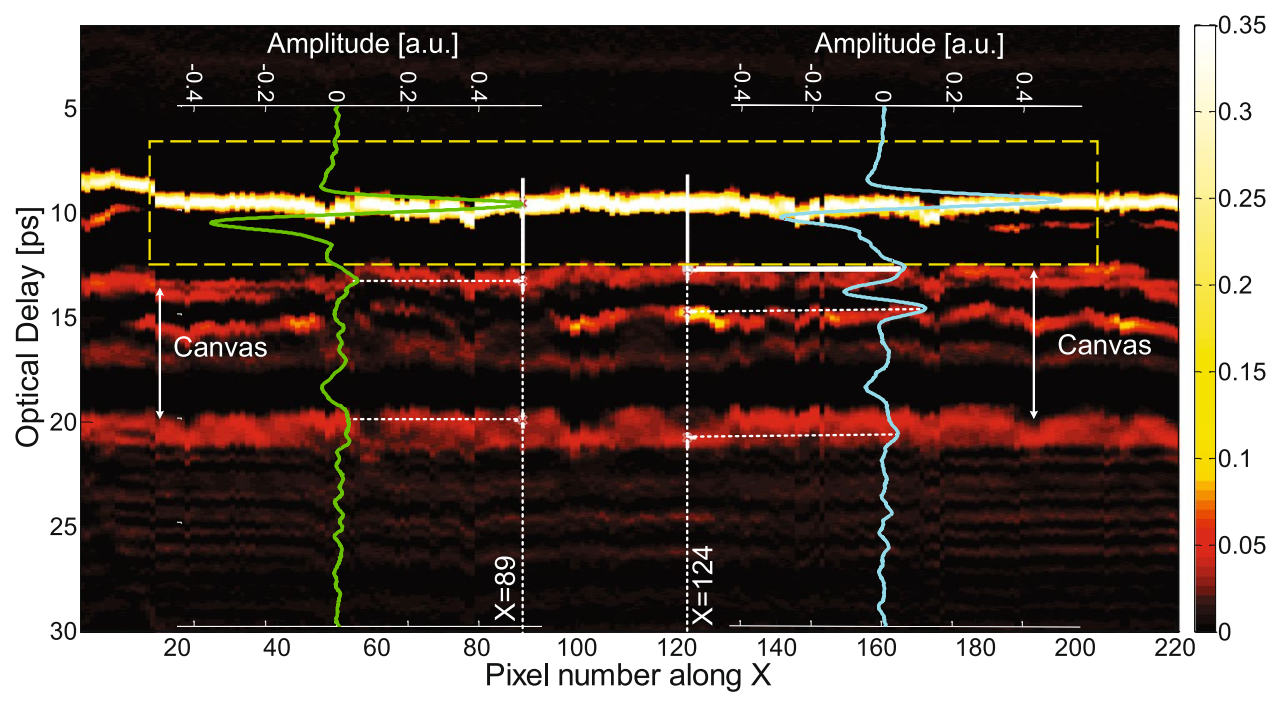

Figure 3. The THz B-scan based on the amplitude of raw signals with the cross-section $Y=150$. Two typical $\mathrm{THz}$ reflected signals are plotted corresponding to the $\mathrm{THz}$ B-scan, with the waveform at pixel $(89,150)$ in green and the waveform at pixel $(124,150)$ in blue.

features at $\sim 15.5$ and 17 ps are due to the fact that the painting contains a double layer of canvas, as is verified by visual inspection of the edge of the painting. The presence of a double-canvas support is often indicative of a remounting and/or restoration of the painting. We also note what appear to be mass-produced wire nails in the stretcher supporting a possible late 19th or 20th century remounting, but no record exists of this. A few pronounced inhomegeneities inside the canvas, which produce additional echoes, are evident in the B-scan and in the C-scan based on the peak-to-valley value in the time slice, in Fig. 2(c) above.

Moving above the canvas into the paint layers (optical delay $<14 \mathrm{ps}$ ), however, the $\mathrm{THz} \mathrm{B}$-scans based on the raw data do not provide any clear stratigraphic detail, since the stratigraphy of the painting above the canvas is hidden in the first cycle of the reflected THz signal (which is blocked in yellow in Fig. 3), and the depth resolution based on the raw $\mathrm{THz}$ signals is not high enough to resolve the various paint layers. The available $\mathrm{THz}$ spectrum in our system extends to $\sim 3 \mathrm{THz}$; this corresponds to $\sim 100 \mu \mathrm{m}$. Accounting for a representative refractive index of $1.85^{10}$, this limits us to a depth resolution, conventionally defined, of $100 \mu \mathrm{m} / 1.85 \approx 54 \mu \mathrm{m}$. In other words, the absence of useful information from the raw $\mathrm{THz}$ is not merely due to the overlapping echoes; the minimum optical thickness that can be resolved is limited to $\sim 100 \mu \mathrm{m}$. Nonetheless, as we shall see, due to the nature of the painting's layer structure, we can reconstruct the stratigraphy on a scale significantly below $\sim 100 \mu \mathrm{m}$. This is also the reason the time-domain $\mathrm{THz}$ C-scan in Fig. 2(a) contains both the surface and subsurface features.

Sparsity-based Deconvolved Signals. As mentioned above, the detailed stratigraphy associated with the painting itself is not evident in the raw data. Nonetheless, the sought for information is contained in the data; it is a matter how to extract it in order to reconstruct the stratigraphy. Sparsity-based time-domain deconvolution based 

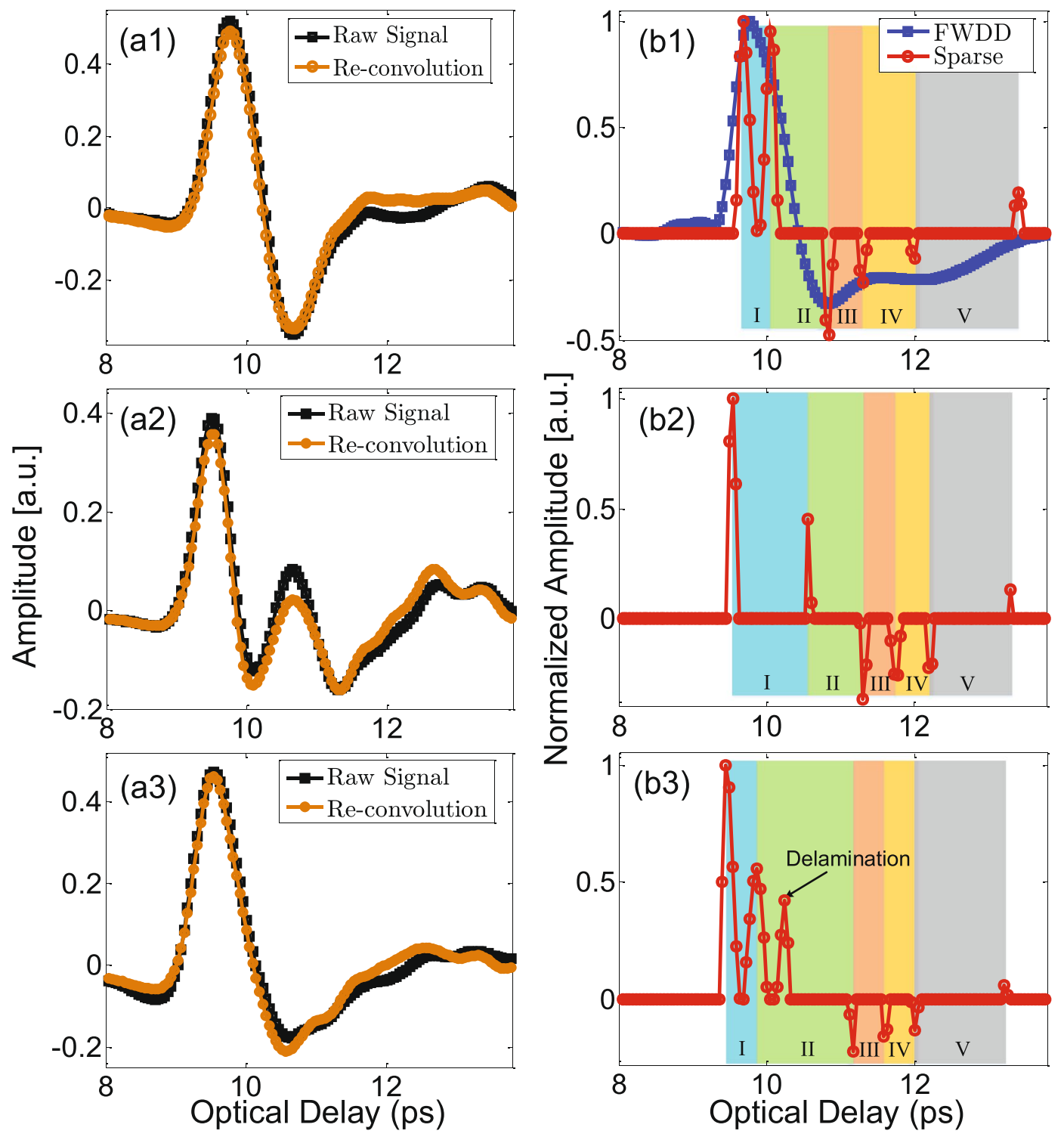

Figure 4. Typical THz reflected signals and the sparsity-based deconvolved signals. Figure 4(a1-a3) show examples of raw reflected signals (black) and signals reconstructed (brown) from the sparse-deconvolution and reference signals. Figure 4(b1-b3) show the corresponding sparsity-based deconvolution signals (red); Fig. 4(b1) also shows the deconvolved signal achieved by FWDD for comparison.

on the shrinkage algorithm is utilized to process the 3D volume raw data. After deconvolution, a sparsity-based impulse-response function is achieved, which entirely depends on the stratigraphy and provides a new imaging domain with enhanced depth resolution. In the left-hand frames are shown three examples of raw $\mathrm{THz}$ signals received at various pixels (black curves) in Fig. 4(a1-a3). The corresponding sparsity-based impulse response functions are shown in the right-hand frames, Fig. 4(b1-b3). FWDD (detailed procedure in ${ }^{26}$ ) is also employed to process the raw data for comparison. In the deconvolution process, we consider the $\mathrm{THz}$ reference signal as the input and the reflected $\mathrm{THz}$ signal as the output; therefore, the actual impulse-response function associated with reflection coefficients should be obtained by multiplying the deconvolved signal by a factor of -1 for phase correction.

The sparsity-based deconvolution result of the reflected THz signal at pixel $(89,150)$ is shown in Fig. 4(b1). Six peaks are clearly identified in the sparsity-based impulse-response function, which correspond to five separated layers above the canvas. Compared with the result achieved by FWDD, which presents quite broad peaks and fails to reveal the underlying stratigraphy, the sparsity-based deconvolution result shows sharp features associated with interfaces between optically thin layers. Based on a knowledge of the typical structure of easel paintings $s^{27}$ of this period and visual inspection (see below), these five layers are expected to correspond to the varnish, pictorial layer, underpainting layer, imprimatura, and ground layer. The peaks are positive or negative, depending on whether the refractive index of the following layer is larger or less than that of the preceding layer. Finally, to check that the sparsity-based deconvolved signals indeed correspond to our raw signals, we convolve the deconvolved signals with the $\mathrm{THz}$ reference signal obtained by reflecting off a metal plate, which is referred to 


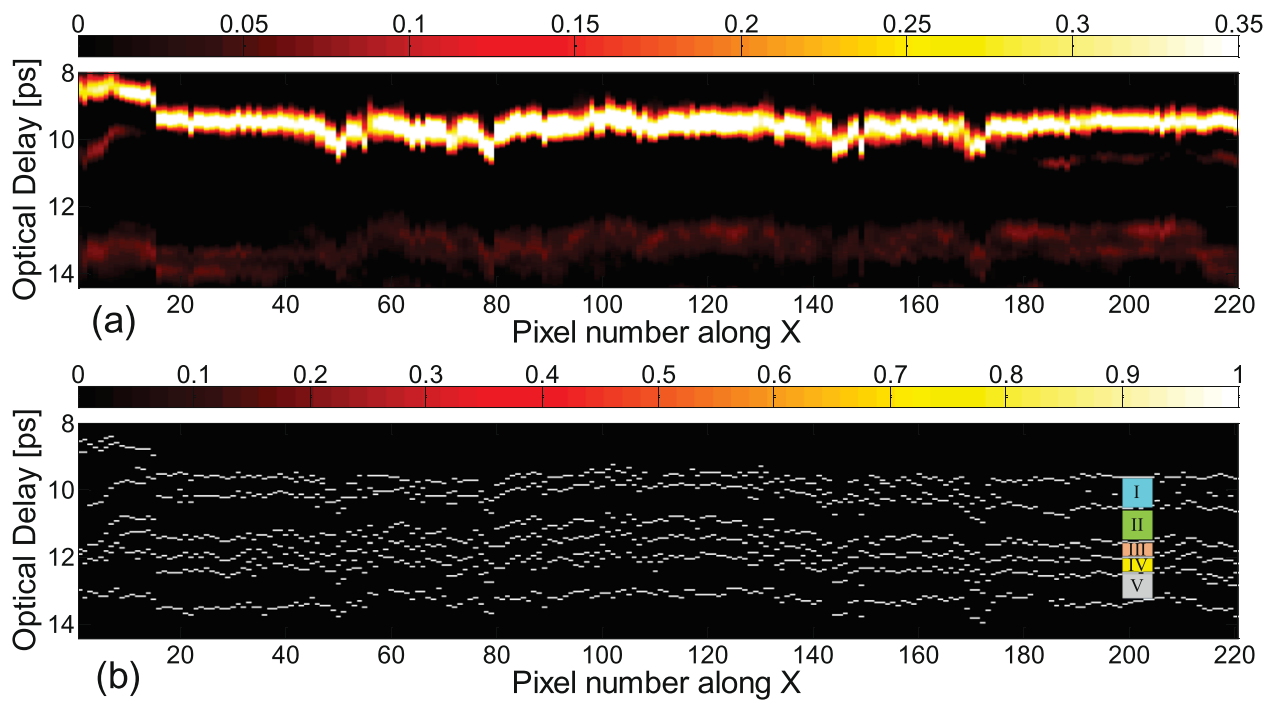

Figure 5. Comparison for optical delay corresponding to the layers above the canvas between (a) the $\mathrm{THz}$ B-scan based on the raw data (Fig. 3 on an expanded vertical scale) and (b) the binary THz B-scan based on the deconvolved data with the cross-section $Y=150$ in which a valid peak is assigned value ' 1 ' and the other positions ' 0 ' regardless of the sign or height of the peak. I: varnish; II: pictorial layer; III: underpainting; IV: imprimatura; V: ground layer.

as "re-convolution", and recover reconstructed signals [brown curves, Fig. 4(a1-a3)] with high fidelity compared with the received raw signals. This verifies our sparsity-based deconvolution procedure.

The sparsity-based impulse-response function, which consists of a baseline at zero and then a sequence of sharp peaks, enables us to reconstruct the detailed stratigraphy of the painting. By performing a peak-detection (both positive and negative peaks), binary THz B-scans, in which a valid peak is assigned value ' 1 ' and the other positions ' 0 ' regardless of the sign or height of the peak, can be obtained. The binary THz B-scan with the cross-section $Y=150$ is shown in Fig. 5(b). The comparison with the THz B-scan based on the raw signals in Fig. 5(a), shows that the binary THz B-scan unlocks a wealth of information absent in the raw B-scan. It reveals, for the first time based on THz reflectometry, the detailed stratigraphy above the supporting canvas of a pre-19th century easel painting.

Reconstruction of the Stratigraphy. In this section we synthesize our foregoing results to provide a tentative account of the stratigraphy of Madonna in Preghiera. The sparsity-based deconvolved signal, as seen above, contains a wealth of information concerning the various layers present in the painting as seen in the B-scan of Fig. 5. In order to reconstruct the layer-structure across the painting, it is useful to present the deconvolved data as C-scans that emphasize individual layers. This can be done by focusing on the amplitude of a specific reflected peak or the optical delay between specific successive peaks in the signals obtained across the canvas.

$\mathrm{THz} \mathrm{C}$-scans based on the amplitude of the first reflected peak and C-scans based on the optical delay between the first and the second peaks in the deconvolved signals are shown in Fig. 6, which reveal the features of the topmost layer in the painting, viz, the varnish. The THz C-scan in Fig. 6(a), shows only faint features associated with the painting's composition. Instead, the C-scan is dominated by the morphology of the varnish surface, i.e., the surface roughness and variations associated with the craquelure due to aging. The lighter, more yellow areas to the left and right of the Madonna's head indicate a smoother surface morphology in these regions. The THz C-scan in Fig. 6(b), based on optical delay between the first two reflected peaks, provides the quantitative information about the varnish thickness across the painting. Surface-morphology-related features are somewhat suppressed as they do not have a major effect on the varnish thickness across the painting. Based on this C-scan, we observe that the varnish around the Madonna's profile is thicker to the left and right of her head--the same areas indicating a higher-amplitude first reflected peak. Another striking point is that a small region near the right of the Madonna's head, as well as the left edge of the painting, shows much thicker varnish. Note that these features seen in the two $\mathrm{C}$-scans discussed above are also evident in the raw and sparsity-based deconvolved signals. At representative pixel $(185,158)$, the optical delay between the first and second peak is larger, as shown in Fig. 4(b2).

The THz C-scans based on the amplitudes of the second and third peaks, corresponding to the varnish/pictorial and pictorial/underpainting interfaces, are plotted in Fig. 7(a,b). On the one hand, Fig. 7(a) clearly shows the painting's composition, and is due to the pigment-dependent refractive index of the various colors evident in the final composition. On the other hand, in Fig. 7(b) the composition, while visible is less pronounced than in Fig. 7(a). This is due to the likelihood that the first-applied pictorial layer may have been fairly uniform in color as was common practice; the most prominent features of the composition, such as drapery, facial features, and hands, were painted subsequently. The various individual paint applications in the pictorial layer thus involve fairly gradual changes in refractive index in layers too thin to resolve in our measurements. Also note that a fine network of horizontal and vertical lines is evident. The optical delay between the second and third peaks 


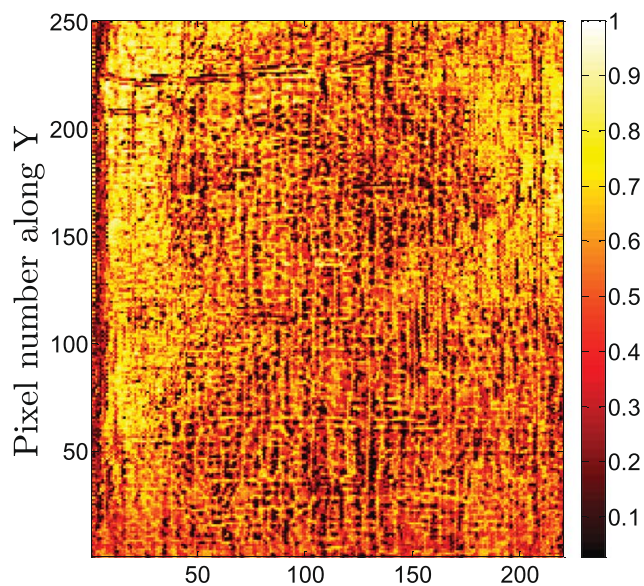

(a) Pixel number along $\mathrm{X}$

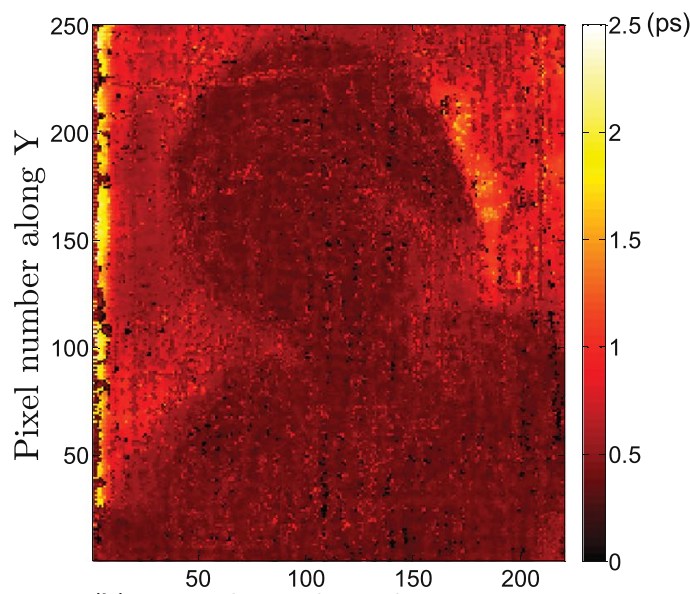

(b) Pixel number along $\mathrm{X}$

Figure 6. Varnish features revealed by THz sparsity-based deconvolved signals. (a) THz C-scan based on the amplitude of the first peak of the deconvolved signal. The contrast is normalized to one; (b) THz C-scan based on the optical delay between the first and second peaks of the deconvolved signal.

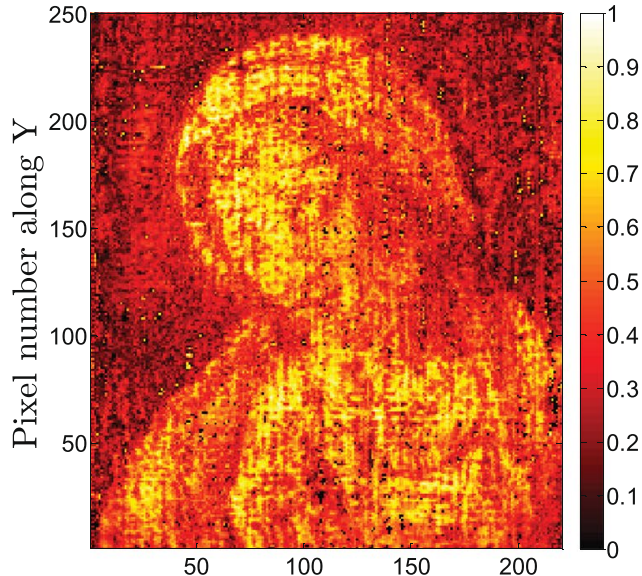

(a) Pixel number along $\mathrm{X}$

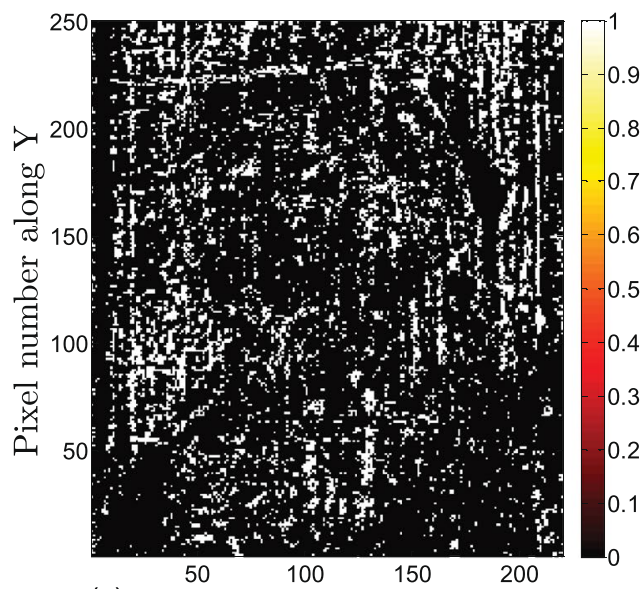

(c) Pixel number along $\mathrm{X}$

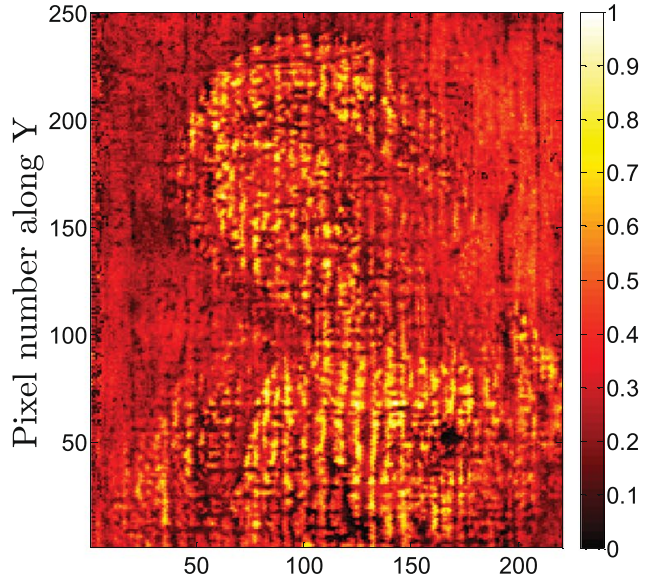

(b) Pixel number along $\mathrm{X}$

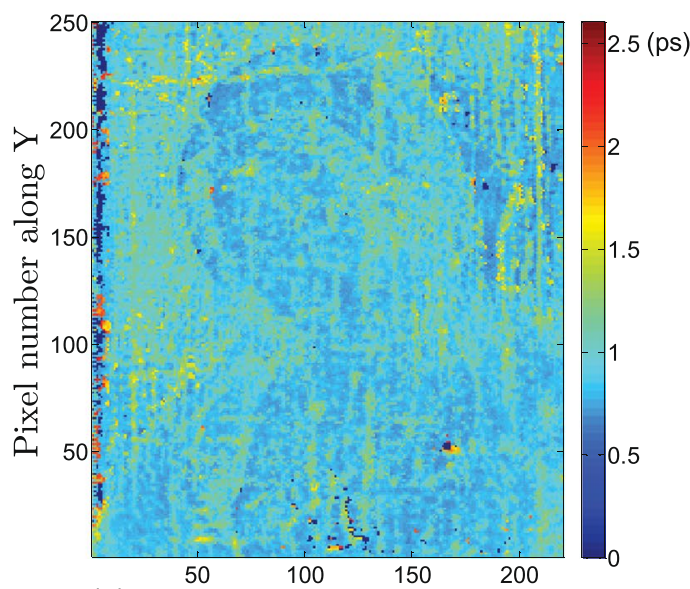

(d) Pixel number along $\mathrm{X}$

Figure 7. Features of the pictorial layer revealed by $\mathrm{THz}$ sparsity-based deconvolved signals. (a) THz C-scan based on the amplitude of second peak of the deconvolved signals; (b) THz C-scan based on the amplitude of the peak corresponding to the interface between the pictorial and underpainting layers; (c) binary THz C-scan indicating the positions where delamination occurs; (d) $\mathrm{THz} \mathrm{C}$-scan based on the optical delay between the peaks corresponding to the thickness of the pictorial layer. 


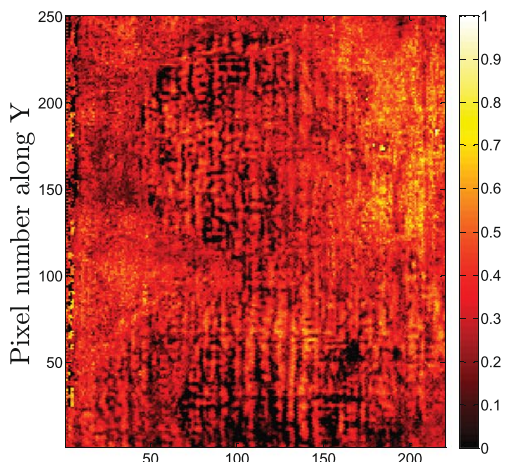

(a1) Pixel number along $\mathrm{X}$

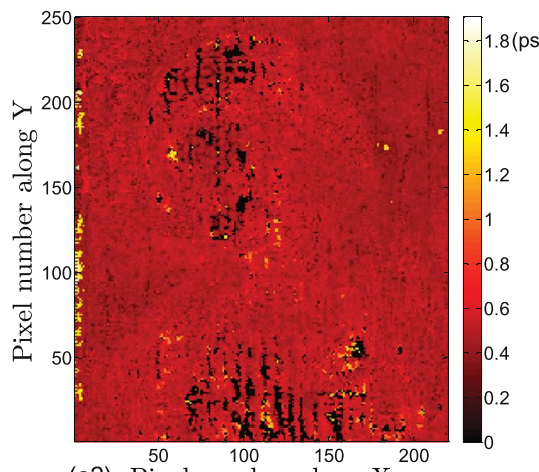

(a2) Pixel number along $X$

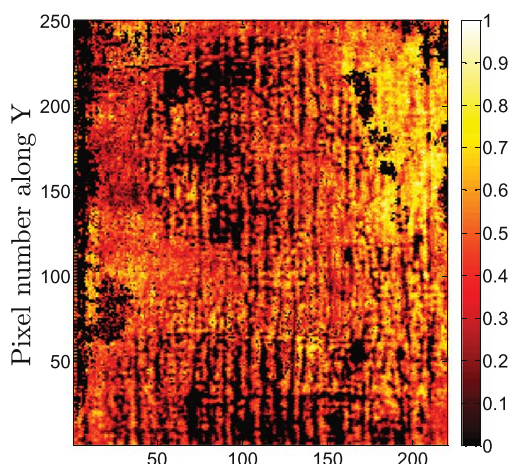

(b1) Pixel number along $\mathrm{X}$

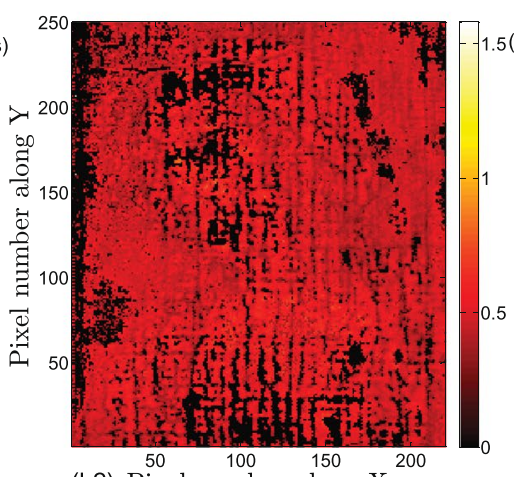

(b2) Pixel number along $X$

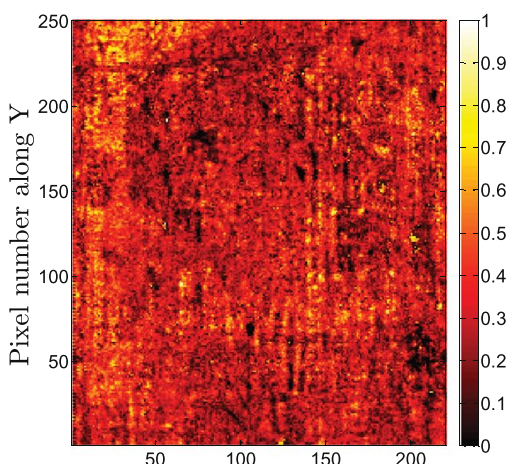

(c1) Pixel number along $X$

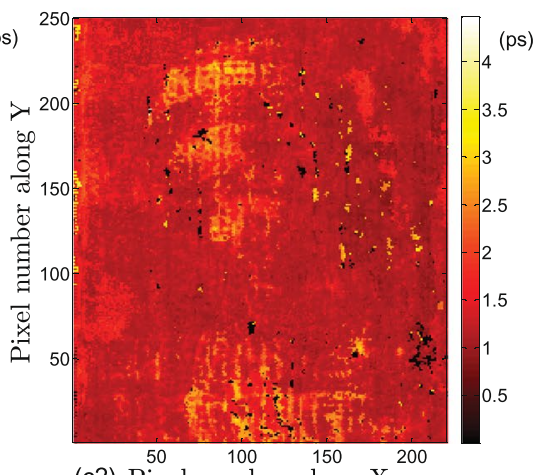

(c2) Pixel number along $\mathrm{X}$

Figure 8. Features of the underpainting, imprimatura, and ground layers revealed by THz C-scans based on sparsity-based deconvolved signals. THz C-scans based on the amplitude of (a1) the fourth, (b1) fifth, and (c1) sixth peak. Contrast is normalized to one. $\mathrm{THz}$ C-scans based on the optical delay between (a2) the third and fourth peaks, (b2) fourth and fifth peaks, and (c2) fifth and sixth peaks.

in the deconvolved signals provides information about the optical thickness of the pictorial layer; however, the features are not uniform across the painting, shown in Fig. 7(d), and the horizontal and vertical lines are more pronounced. These lines are associated with the age-induced craquelure, where an addition peak between the second and the third peaks can be detected in the corresponding sparsity-based deconvolved signal. A typical signal illustrating this at pixel $(112,229)$ (a typical pixel in the horizontal line) is shown in Fig. 4(a3,b3). The physical origin of this peak is the existence of an additional air gap in these areas; however, this layer is too thin to be resolved even in the deconvolved signal, which generates only one observable positive peak. We summarize the presence of this additional peak, and thus delaminated regions, in the binary THz C-scan in Fig. 7(c). In this image, white regions indicate the presence of the additional peak, black its absence. The white regions are highly correlated with the craquelure; this is not entirely surprising, since stresses associated with craquelure formation and the channels open to the infiltration of moisture and other contaminants may also lead to delamination localized there.

Continuing to consider subsequent peaks in the sparsity-based deconvolved signal, features associated with the underpainting, imprimatura, and ground layers are investigated based on the THz C-scans in Fig. 8. Figure 8(a1-a3) provide C-scans based on the amplitudes of the fourth, fifth, and sixth peaks, respectively. Lines associated with the craquelure are still evident due to the shadow effects associated with reduced signals reaching subsequent layers depending on $\mathrm{THz}$ reflectivity and scattering from layers above. We note that the fourth and fifth peaks cannot be resolved in the deconvolved signals in all the pixels across the painting, corresponding to the black regions in the $\mathrm{THz}$ C-scans. Most of the black regions appear in the woman's profile. This might be due to the multiple paint applications in the profile, which makes the layers underneath thinner than the depth resolution achieved. In Fig. 8(b1-b3) are shown C-scans computed from the optical delay between the third and fourth, the fourth and fifth, and the fifth and sixth peaks, respectively, if resolved. We note that, for Fig. 8(c), the optical delay is calculated based on the peak corresponding to the ground/canvas interface and the preceding peak which can be resolved. Based on the results, we conclude that the Master of the Madonna in Preghiera applied layers of fairly uniform thickness.

\section{Discussion}

Sparsity-based THz reflectometry provides detailed 3D information on the 10- $\mu m$-to- $m m$ scale in the depth direction and the 100-of- $\mu m$-to- $m$ scale in the transverse directions, enabling us to reconstruct the detailed stratigraphy of the Madonna in Preghiera. Quantitative information in depth, such as the physical thicknesses of each resolved layer, can be estimated based on the knowledge of corresponding refractive indices. Starting with the canvas, a ground layer (gesso) is applied to seal the canvas and to create a smooth surface on which to paint. The 


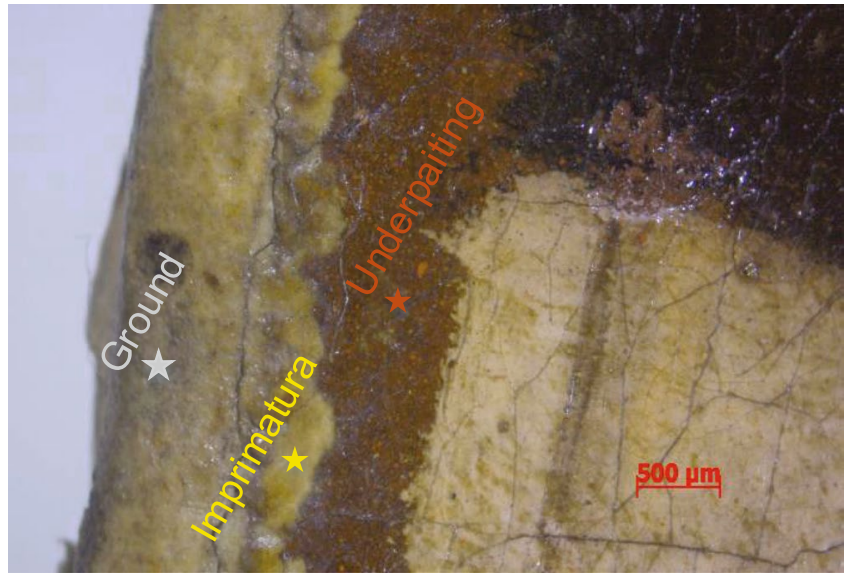

Figure 9. The optical microscopic image of a small region at the edge of Madonna in Preghiera. The applications of ground, imprimatura, and the underpainting can be clearly identified.

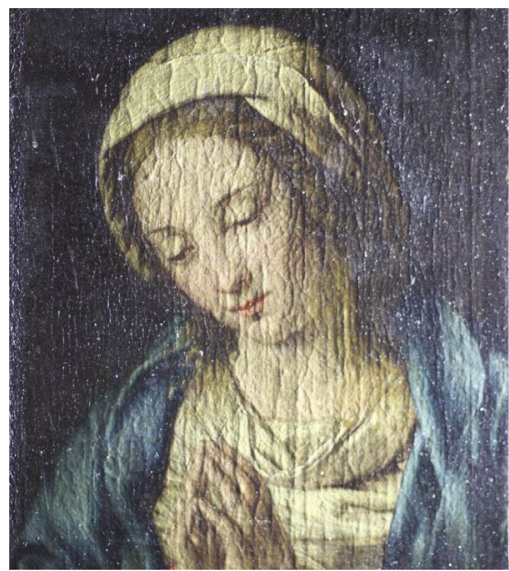

(a)

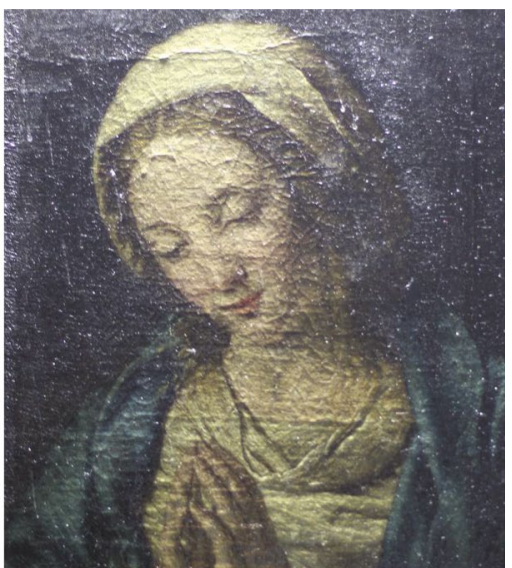

(b)

Figure 10. White-light raking images of Madonna in Preghiera with the light source (a) placed on the right side and $(\mathbf{b})$ the top side of the painting.

imprimatura, literally the 'first paint layer,' follows the ground that would seal the oil-absorbent layer. Without the imprimatura, paint directly applied would soak into the ground and be difficult to control. The underpainting layer provides a proper foundation of the scene/subject matter, which was typically painted in a dark and muted monochrome tone, usually consisting of umber, as brown underpainting has often be used in oil painting from the 15 th to 17 th centuries. Such an approach was widespread to the point that the entire tonality and compositions of paintings frequently accounted for this dark underpainting. THz B- and C-scans clearly reveal the features of the ground, imprimatura, and the underpainting layers, which are fairly uniform across the painting. Assuming the refractive index of gesso is about $1.52^{10}$, the average physical thickness of the ground layer is about $132.7 \mu \mathrm{m}$. For other oil-based paint layers, although the difference of refractive index between each layer is sufficient to produce the $\mathrm{THz}$ reflections, we assume a mean refractive index of $1.85^{10}$ in order to estimate the physical thickness of each paint layer. Based on this assumed refractive index, we estimate the average thickness of imprimatura is $\sim 34 \mu \mathrm{m}$ and the average thickness of the underpainting layer is $\sim 38 \mu \mathrm{m}$. The presence of these three preparatory layers is confirmed by optical microscopy near the painting's edge, shown in Fig. 9, where these layers are exposed.

The pictorial layers are applied subsequent to the underpainting. They contain the visually evident composition of the finished painting, and consist of additional applications of background color, the figure of the Madonna, facial features, hands, the gossamer veil, and other fine details. In the $\mathrm{THz} \mathrm{C}$-scans, textural features due to the craquelure first become pronounced in the pictorial layer. This is also where we observe the presence of delamination, closely associated with the craquelure. The surface morphology due to the craquelure can also be seen in white-light raking images shown in Fig. 10. In Fig. 10(a), the raking light source was placed to the right of the painting, emphasizing inhomogeneities oriented in the vertical direction; in Fig. 10(b), the raking light source was placed toward the top side of the painting, emphasizing inhomogeneities oriented in the horizontal direction. Again, there is strong correlation of the features seen in the raking-light images and in the THz C-scans in Fig. 7. 
Based on the assumed refractive index 1.85, the average physical thickness of the pictorial layer is about $64 \mu \mathrm{m}$ in the regions without craquelure.

It was typical in the 17th century finally to apply varnish (a natural resin) to saturate the paint colors and protect the surface. The craquelure continues to be pronounced in the varnish, as is evidenced in the corresponding $\mathrm{THz}$ C-scans. The application of the varnish is not uniform across the painting. Assuming the refractive index of the varnish is about $1.6^{28}$, the physical thickness of the varnish at pixel $(89,150)$ is about $35 \mu \mathrm{m}$. It is important to note that the thicker region near the Madonna's head is clearly identified, which exhibits the same shape as the dark areas in the UV fluorescence image in Fig. 1(c). The physical thickness at pixel $(185,158)$ is estimated as $96 \mu \mathrm{m}$. This thick region is identified as a retouchings of the varnish. Visual inspection shows that these regions exhibit an anomalous texture.

The depth resolution of THz reflectometry is greatly enhanced by exploiting sparsity-based deconvolution. Indeed, conventional deconvolution methods, such as FWDD, entirely fail to reveal the underlying stratigraphy in easel paintings, because FWDD actually involves filtering out low- and high-frequency noise introduced from the division operation in the conventional deconvolution process, and therefore, narrows the bandwidth of the resulting impulse-response function ${ }^{29}$. Sparsity-based deconvolution is a pure time-domain method which does not introduce the low- and high-frequency noise, and is able to yield an enhanced depth resolution and achieve a more clear representation of the stratigraphy. In addition, sparsity-based deconvolution appears to have a better dynamic range than FWDD which implies that it is more effective in resolving small echoes. This property is especially important, since the difference of refractive index between various layers is often small in art paintings. We note that the depth resolution achieved by sparsity-based deconvolution is fundamentally limited by the coherence length of the $\mathrm{THz}$ source and the signal-to-noise ratio during the measurement.

In summary, sparsity-based $\mathrm{THz}$ reflectometry presented in this study clearly revealed the detailed stratigraphy of a 17th century easel painting with layer-thicknesses less than $50 \mu \mathrm{m}$, including the varnish, pictorial, underpainting, imprimatura, and the ground layers across the entire painting. Retouching of varnish, as well as age-induced craquelure in the pictorial layer are also successfully characterized. The results achieved by sparsity-based $\mathrm{THz}$ reflectometry are supported by other techniques. We have demonstrated that sparsity-based $\mathrm{THz}$ reflectometry promises to provide an effective in-situ 3D quantitative imaging modality with high depth resolution and dynamic range for a broad range of cultural heritage objects, and an invaluable contribution to art-historical studies, as well as for conservation, restoration, and authentication.

\section{Methods}

THz reflective imaging. A typical THz time-domain system (Teraview TPS Spectra 3000) is employed in this study. The GaAs photoconductive antenna is excited by an ultrafast laser to produce roughly single-cycle $\mathrm{THz}$ pulses with bandwidth extending from $60 \mathrm{GHz}$ to $3 \mathrm{THz}$. The maximum peak of its power spectrum is located at about $0.3 \mathrm{THz}$. The focus spot size of the THz beam is frequency-dependent, and is about $300 \mu \mathrm{m}$ at $1 \mathrm{THz}$. Each recorded temporal reflected $\mathrm{THz}$ waveform contains 1024 data points, and the data sampling period is set to $0.0465 \mathrm{ps}$. The signal is averaged over 10 shots per pixel to enhance signal to noise. The scanning of the painting was conducted in a temperature-controlled laboratory at $22^{\circ} \mathrm{C}$. The humidity in the laboratory was held about $38 \%$.

Sparsity-based Time-Domain Deconvolution. In the time domain, the THz reflected signal (electric field) $r(t)$ is the convolution of the incident $\mathrm{THz}$ pulse $i(t)$ with the impulse-response function $h(t)$, which corresponds to the structure and properties of the sample at a given point of interest,

$$
r(t)=i(t) \otimes h(t)=\int_{-\infty}^{+\infty} i(\tau) h(t-\tau) d \tau .
$$

For reflective THz imaging, the incident $\mathrm{THz}$ pulse $i(t)$ can be obtained by first recording the $\mathrm{THz}$ signal reflected from a metal plate ( $\mathrm{THz}$ reference signal), and then multiplying the reference signal by a factor of -1 for phase correction. In practice, we should consider the discrete form of (1) with the sampling period $T_{s}$,

$$
r_{n}=\sum_{m=0}^{M-1} i_{m} h_{n-m}+e_{n},
$$

where $r_{n}=r\left(n T_{s}\right), i_{m}=i\left(m T_{s}\right)$ and $e_{n}$ accounts for the noise originating from the measurement system and materials with $n$ and $m$ as the indices of data points, and $M$ as the length of the data points. Let column vectors $\mathbf{r}, \mathbf{i}, \mathbf{h}$ and $\mathbf{e}$ collect the samples of $r_{n}, i_{n}, h_{n}$ and $e_{n}$, respectively. Then (2) can be expressed as

$$
\mathbf{r}=\mathbf{A h}+\mathbf{e},
$$

where $\mathbf{A}$ is the convolution matrix whose columns are delayed versions of $\mathbf{i}$.

The basic idea of sparse deconvolution is to achieve the impulse response function by exploiting the sparse constraint. It aims at approximating the received $\mathrm{THz}$ signal $\mathbf{r}$ with $\mathbf{A h}$ where $\mathbf{h}$ is a sparse sequence; that is, $\mathbf{h}$ has only few non-zero components. In this case, the sparse vector $\mathbf{h}$ can be computed by solving the $l_{0}$ regularized optimization problem, which is defined as

$$
\min _{\mathbf{h}} \frac{1}{2}\|\mathbf{A h}-\mathbf{r}\|_{2}^{2}+\lambda\|\mathbf{h}\|_{0},
$$


where $\|\mathbf{h}\|_{0}$ is the $l_{0}$-norm of $\mathbf{h}$, which is defined to be the number of nonzero entries in $\mathbf{h}$, and $\lambda$ is the regularization parameter, which controls the tradeoff between the sparsity of $\mathbf{h}$ and the residue norm. However, solving the non-convex $l_{0}$ regularized optimization problem is known to be nonpolynomial hard and the global optimum cannot be guaranteed. It has already been shown that this non-convex optimization problem can be approximated with a convex optimization problem by replacing the $l_{0}$ penalty with the $l_{1}$ penalty as

$$
\min _{\mathbf{h}} \frac{1}{2}\|\mathbf{A h}-\mathbf{r}\|_{2}^{2}+\lambda\|\mathbf{h}\|_{1}
$$

where $\|\mathbf{h}\|_{1}$ is the $l_{1}$-norm of $\mathbf{h}$, which is defined as the sum of the absolute values of its components. Since the $l_{1}$-norm is convex, a global optimum can be guaranteed. An iterative shrinkage algorithm is utilized in this study because of its effectiveness and the limited number of parameters that need to be tuned. The general iterative procedure is given by:

$$
\mathbf{h}_{i+1}=S_{\lambda \tau}\left(\mathbf{h}_{i}-\tau \mathbf{A}^{\mathrm{T}}\left(\mathbf{A} \mathbf{h}_{i}-\mathbf{r}\right)\right)
$$

where $\tau$ is an appropriate step size, which should obey

$$
\tau<\frac{2}{\left\|\mathbf{A}^{\mathrm{T}} \mathbf{A}\right\|_{2}}
$$

in order to guarantee convergence, and the shrinkage or soft-thresholding operator $S_{\lambda \tau}$ is defined as

$$
S_{\lambda \tau}(h[n])= \begin{cases}h[n]+\lambda \tau & h[n] \leq-\lambda \tau \\ 0 & |h[n]|<\lambda \tau \\ h[n]-\lambda \tau & h[n] \geq \lambda \tau\end{cases}
$$

A thorough theoretical analysis ${ }^{30}$ proves the convergence of this iterative shrinkage algorithm guaranteeing that the solution is the global minimizer for convex $\mathbf{h}$. For processing the raw $3 \mathrm{D}$ volume $\mathrm{THz}$ data from the painting in this study, the iteration based on Eq. 6 was performed 3000 times with the regulation parameter $\lambda=0.4$ and the step size 1.2/ $\left\|\mathbf{A}^{\mathrm{T}} \mathbf{A}\right\|_{2}$. Detailed information on the accuracy of the algorithm can be found in our previous results ${ }^{24}$.

Data Availability. The datasets generated during and/or analysed during the current study are available from the corresponding author on reasonable request.

\section{References}

1. Reischig, P. et al. High-resolution non-invasive 3D imaging of paint microstructure by synchrotron-based X-ray laminography. Appl. Phys. A 111, 983-995 (2013).

2. Reiche, I. et al. Depth profiling reveals multiple paint layers of Louvre Renaissance paintings using non-invasive compact confocal micro-X-ray fluorescence. J. Anal. At. Spectrom. 27, 1715 (2012).

3. Villafana, T. E. et al. Femtosecond pump-probe microscopy generates virtual cross-sections in historic artwork. Proc. Natl. Acad. Sci. 111, 1708-1713 (2014).

4. Presciutti, F. et al. Noninvasive nuclear magnetic resonance profiling of painting layers. Appl. Phys. Lett. 93, 33505 (2008).

5. Cheung, C. S., Spring, M. \& Liang, H. Ultra-high resolution Fourier domain optical coherence tomography for old master paintings. Opt. Express 23, 10145 (2015).

6. Alfeld, M. \& Broekaert, J. A. C. Mobile depth profiling and sub-surface imaging techniques for historical paintings-A review. Spectrochim. Acta Part B At. Spectrosc. 88, 211-230 (2013).

7. Duling, I. \& Zimdars, D. Terahertz imaging: Revealing hidden defects. Nat. Photonics 3, 630-632 (2009).

8. Jackson, J. B. et al. A Survey of Terahertz Applications in Cultural Heritage ConservationScience. IEEE Trans. Terahertz Sci. Technol. 1, 220-231 (2011).

9. Seco-Martorell, C. et al. Goyass artwork imaging with Terahertz waves. Opt. Express 21, 17800-17805 (2013).

10. Gomez-Sepulveda, A. M. et al. History of Mexican Easel Paintings from an Altarpiece Revealed by Non-invasive Terahertz TimeDomain Imaging. J. Infrared, Millimeter, Terahertz Waves 38, 403-412 (2017).

11. Koch-Dandolo, C. L., Filtenborg, T., Fukunaga, K., Skou-Hansen, J. \& Jepsen, P. U. Reflection terahertz time-domain imaging for analysis of an 18th century neoclassical easel painting. Appl. Opt. 54, 5123-5129 (2015).

12. Tonouchi, M. Cutting-edge terahertz technology. Nat. Photonics 1, 97-105 (2007).

13. Jepsen, P. U., Cooke, D. G. \& Koch, M. Terahertz spectroscopy and imaging - Modern techniques and applications. Laser Photon. Rev. 5, 124-166 (2011).

14. Picollo, M., Fukunaga, K. \& Labaune, J. Obtaining noninvasive stratigraphic details of panel paintings using terahertz time domain spectroscopy imaging system. J. Cultural Heritage 16, 73-80 (2015).

15. Koch-Dandolo, C. L. \& Jepsen, P. U. Wall Painting Investigation by Means of Non-invasive Terahertz Time-Domain Imaging (THzTDI): Inspection of Subsurface Structures Buried in Historical Plasters. J. Infrared, Millimeter, Terahertz Waves 37, 198-208 (2016).

16. Koch-Dandolo, C. L., Picollo, M., Cucci, C. \& Jepsen, P. U. Non-invasive Florentine Renaissance Panel Painting Replica Structures Investigation by Using Terahertz Time-Domain Imaging (THz-TDI) Technique. J. Infrared, Millimeter, Terahertz Waves 37, 1148-1156 (2016).

17. Koch-Dandolo, C. L. et al. Insights on the Side Panels of the Franciscan Triptych by Fra Angelico Using Terahertz Time-Domain Imaging (THz-TDI). J. Infrared, Millimeter, Terahertz Waves 38, 413-424 (2017).

18. Fukunaga, K. THz Technology Applied to Cultural Heritage in Practice. Cultural Heritage Science (Springer Japan, 2016).

19. Adam, A. J. L., Planken, P. C. M., Meloni, S. \& Dik, J. Terahertz imaging of hidden paint layers on canvas. Opt. Express 17, 3407-3416 (2009).

20. Redo-Sanchez, A. et al. Terahertz time-gated spectral imaging for content extraction through layered structures. Nat. Commun. 7, 12665 (2016).

21. Walker, G. C. et al. Terahertz deconvolution. Opt. Express 20, 27230-27241 (2012).

22. Chen, Y., Huang, S. \& Pickwell-MacPherson, E. Frequency-wavelet domain deconvolution for terahertz reflection imaging and spectroscopy. Opt. Express 18, 1177-1190 (2010). 
23. Dong, J. et al. Terahertz frequency-wavelet domain deconvolution for stratigraphic and subsurface investigation of art painting. Opt. Express 24, 26972 (2016).

24. Dong, J., Wu, X., Locquet, A. \& Citrin, D. S. Terahertz Superresolution Stratigraphic Characterization of Multilayered Structures Using Sparse Deconvolution. IEEE Trans. Terahertz Sci. Technol. 7, 260-267 (2017).

25. Dondi, P., Lombardi, L., Malagodi, M. \& Licchelli, M. Automatic identification of varnish wear on historical instruments: The case of Antonio Stradivari violins. J. Cult. Herit. 22, 968-973 (2016).

26. Dong, J., Locquet, A. \& Citrin, D. S. Terahertz Quantitative Nondestructive Evaluation of Failure Modes in Polymer-Coated Steel. IEEE J. Sel. Top. Quantum Electron. 23, 1-7 (2017).

27. Nevin, A., Pouli, P., Georgiou, S. \& Fotakis, C. Laser conservation of art. Nat. Mater. 6, 320-322 (2007)

28. Yun-Sik, J., Geun-Ju, K. \& Seok-Gy, J. Terahertz Dielectric Properties of Polymers. Journal of Korean Physical Society 49, S. 513 (2006).

29. Dong, J., Locquet, A. \& Citrin, D. S. Depth resolution enhancement of terahertz deconvolution by autoregressive spectral extrapolation. Opt. Lett. 42, 1828 (2017).

30. Daubechies, I., Defrise, M. \& De Mol, C. An iterative thresholding algorithm for linear inverse problems with a sparsity constraint. Commun. Pure Appl. Math. 57, 1413 (2004).

\section{Acknowledgements}

The authors thank Anne Adrian, Claire Meunier, Kévin Kazek, and Philippe Brunella of the Musée de la Cour d'Or-Metz Métropole for the kind loan of the painting and for sharing their expertise in art history and technique. The authors gratefully acknowledge the financial support of the Conseil Régional du Grand Est of the Fonds Européen de Développement Régional (FEDER), and of the Institut Carnot ARTS.

\section{Author Contributions}

J.D. performed the THz measurement; M.M. performed the multispectral imaging experiment; J.D. designed the algorithm and processed the data; D.S.C., J.D., and A.L. wrote the paper. All authors analyzed the results and reviewed the manuscript.

\section{Additional Information}

Competing Interests: The authors declare that they have no competing interests.

Publisher's note: Springer Nature remains neutral with regard to jurisdictional claims in published maps and institutional affiliations.

(c) (i) Open Access This article is licensed under a Creative Commons Attribution 4.0 International License, which permits use, sharing, adaptation, distribution and reproduction in any medium or format, as long as you give appropriate credit to the original author(s) and the source, provide a link to the Creative Commons license, and indicate if changes were made. The images or other third party material in this article are included in the article's Creative Commons license, unless indicated otherwise in a credit line to the material. If material is not included in the article's Creative Commons license and your intended use is not permitted by statutory regulation or exceeds the permitted use, you will need to obtain permission directly from the copyright holder. To view a copy of this license, visit http://creativecommons.org/licenses/by/4.0/.

(c) The Author(s) 2017 